\title{
Cáncer de piel en Colombia: cifras del Instituto Nacional de Cancerología
}

\author{
Flavia Carolina Pozzobon', Álvaro Enrique Acosta², Juan Sebastián Castillo 3 \\ 1. Médica dermatóloga, magíster en Patología Cutánea Avanzada, Clínica de Piel, Instituto Nacional de Cancerología, Bogotá, D.C., \\ Colombia \\ 2. Médico dermatólogo oncólogo, Clínica de Piel, Instituto Nacional de Cancerología; profesor titular, Universidad Nacional de \\ Colombia, Bogotá, D.C., Colombia \\ 3. Médico epidemiólogo, magíster en Salud Pública, investigador Instituto Nacional de Cancerología, Instituto para la Evaluación de \\ la Calidad y Atención en Salud (IECAS, Bogotá, D.C.,
}

\section{RESUMEN}

La incidencia del cáncer de piel ha aumentado en las últimas décadas a nivel mundial. Los datos poblacionales en Colombia son escasos, sin embargo, los registros institucionales de cáncer ofrecen información sobre la frecuencia y la distribución en instituciones de referencia.

En este artículo se resume la información de los diagnósticos nuevos de cáncer de piel correspondientes al período 1996-2010 en el Instituto Nacional de Cancerología, centro de referencia en cáncer a nivel nacional, en donde se evidencia un aumento progresivo de la frecuencia de casos nuevos, acompañado de una mayor frecuencia en los mayores de 75 años.

Entre los subtipos histológicos, predominan el carcinoma basocelular (52,7\%), el escamocelular (22,6 \%) y el melanoma (16,1\%). La incidencia estimada del melanoma maligno en Colombia corresponde a 4,6 por 100.000 en mujeres y 4,4 por 100.000 en hombres.

La importancia del cáncer de piel como prioridad en salud pública, se evidencia en su frecuencia relativa en relación con otros cánceres y en su aparición en todas las etapas de la vida.

PALABRAS CLAVE: neoplasias malignas cutáneas, cáncer de piel, estadísticas médicas, incidencia.

\section{SUMMARY}

Incidence of skin cancer has increased in recent decades worldwide. Population data are limited in Colombia; nevertheless, institutional cancer registries provide information on frequency and distribution of reference institutions.

This article summarizes information of new diagnoses of skin cancer corresponding to the period 1996-2010 at the Instituto Nacional de Cancerología, reference center for cancer in our country, where a progressive increase is evident in frequency of new cases, in addition to an increased frequency in patients older than 75 years.

Basal cell carcinoma (52.7\%), squamous cell (22.6\%) and melanoma $(16.1 \%)$

\section{Correspondencia:}

Flavia Pozzobon

Email:

fcpozzobonnt@unal.edu.co

Recibido: $17 / 10 / 16$

Aceptado: 10/09/17

Conflictos de interés:

No se reportan conflictos de interés.

\section{Financiación:}

Ninguna. 
predominate among histologic subtypes. Colombia's estimated incidence for malignant melanoma corresponds to 4.6/100,000 in women and 4.4/100,000 in men.

The importance of skin cancer as a public health priority is evident in their relative frequency relative to other cancers and their appearance in all stages of life.

KEY WORDS: Cutaneous malignancies, medical statistics, skin neoplasm, incidence

\section{"Existen escasos datos en Colombia sobre el comportamiento epidemiológico de las neoplasias de piel."}

El cáncer de piel es la neoplasia más frecuente del ser humano, y su incidencia continúa creciendo a nivel mundial ${ }^{(1,2)}$. Puede dividirse en dos grandes grupos, según su origen: el melanoma maligno y el cáncer de piel no melanoma. Dentro de este último grupo, el más frecuente es el carcinoma basocelular con una frecuencia de 75 a $80 \%$, seguido por el carcinoma escamocelular, con $15 \%{ }^{(3)}$. El melanoma, aunque hace parte solamente del 3 al 5\% de los casos de cáncer de piel, ocasiona el $75 \%$, aproximadamente, de las muertes por esta entidad ${ }^{(4)}$.

Existen escasos datos en Colombia sobrte el comportamiento epidemiológico de las neoplasias de piel ${ }^{(5,6)}$. El Instituto Nacional de Cancerología, centro de referencia para el manejo del cáncer en Colombia, atendió 6.313 casos nuevos de cáncer - de todas las localizaciones- en el año $2010{ }^{(7)}$, de un total de 70.000 casos estimados incidentes para ese año en el país.

Históricamente, la institución ha dado a conocer el Anuario Estadístico desde el año 1996, en el cual se publican los datos consignados en el Registro Institucional de Cáncer ${ }^{(7)}$, incluyendo la distribución por sexo y grupos etarios de los casos nuevos para todos los tipos de cáncer, cifras seleccionadas de mortalidad institucional, entre otros. Esta herramienta busca orientar la planeación estratégica de las actividades misionales del Instituto.

Se llevó a cabo una revisión de los datos de las fuentes secundarias correspondientes al cáncer de piel en el Instituto Nacional de Cancerología, para el período 1996-2010, con el fin de resumir las frecuencias reportadas para los casos nuevos de cáncer de piel registrados en la institución entre los años 1996 y 2010, así como su variabilidad en el tiempo, y su distribución según edad y sexo.

Para tal fin, se obtuvo una compilación de la estadística institucional procedente de la información consignada en el registro institucional de cáncer y en los anuarios estadísticos para el período de interés ${ }^{(7)}$, teniendo en cuenta los datos correspondientes a los casos nuevos según el diagnóstico histopatológico, la edad, el sexo, el año de diagnóstico y la mortalidad, identificando las frecuencias por año y la media. Se presenta la información en tablas y figuras para facilitar su comprensión.

\section{DATOS DEMOGRÁFICOS Y CASOS NUEVOS DE CÁNCER DE}

\section{PIEL}

Durante el período comprendido entre enero de 1996 y diciembre de 2010, se registraron 79.381 casos nuevos de cáncer en el Instituto Nacional de Cancerología; de estos, 11.780 (14,9\%) fueron casos nuevos de cáncer de piel, con un promedio de 841,4 por año. Sin embargo, al registrar el número de casos nuevos según el subtipo histológico, se encontró un ligero aumento del total de casos, ascendiendo a 11.862 (tabla 1). Esta discrepancia puede ser secundaria a que es posible que exista más de un de cáncer de piel en la misma persona. En el año 2010, de cada 1.000 casos nuevos de cáncer, 202 fueron de piel, en el Instituto Nacional de Cancerología. Se ha observado un incremento notorio de casos nuevos de cáncer de piel atendidos en el Instituto $\mathrm{Na}$ cional de Cancerología (figura 1). En comparación con los otros tipos de cáncer atendidos en el Instituto, se puede evidenciar un incremento sostenido del número de casos de cáncer de piel (23,3 \% en 2010), prácticamente el doble de los de cáncer de mama y de cuello uterino, y de cuatro a cinco veces más que los tumores de la próstata, el sistema hematolinfoide y el sistema gastrointestinal.

La media de edad de los casos de cáncer de piel fue de 62 años. A medida que avanza la edad, aumenta 
Tabla 1. Distribución de casos nuevos de cáncer de piel por diagnóstico histológico, Instituto Nacional de Cancerología, 1996-2010.

\begin{tabular}{|c|c|c|c|c|c|c|c|c|c|c|c|c|c|c|c|c|}
\hline \multicolumn{17}{|c|}{ DISTRIBUCIÓN DE CASOS NUEVOS DE CÁNCER DE PIEL POR DIAGNÓSTICO HISTOLÓGICO, INSTITUTO NACIONAL DE CANCEROLOGÍA, $1996-2010$} \\
\hline SUBTIPO HISTOLÓGICO & 1996 & 1997 & 1998 & 1999 & 2000 & 2001 & 2002 & 2003 & 2004 & 2005 & 2006 & 2007 & 2008 & 2009 & 2010 & Total \\
\hline Basocelular & 395 & 384 & 335 & 221 & 235 & 221 & 313 & 411 & 460 & 523 & 542 & 500 & 506 & 540 & 785 & 6.371 \\
\hline Escamocelular & 145 & 151 & 160 & 116 & 137 & 139 & 164 & 183 & 210 & 219 & 213 & 176 & 174 & 172 & 249 & 2.608 \\
\hline Melanoma & 114 & 100 & 98 & 100 & 84 & 97 & 127 & 162 & 148 & 175 & 124 & 118 & 120 & 143 & 143 & 1.853 \\
\hline Linfoma & 12 & 6 & 17 & 10 & 16 & 18 & 21 & 30 & 38 & 25 & 22 & 20 & 22 & 32 & 38 & 327 \\
\hline Sarcoma de Kaposi & 10 & 13 & 11 & 4 & 6 & 8 & 15 & 13 & 14 & 38 & 12 & 22 & 13 & 9 & 13 & 201 \\
\hline DFSP* & 4 & 5 & 2 & 2 & 3 & 4 & 7 & 9 & 6 & 6 & 1 & 10 & 9 & 5 & 9 & 82 \\
\hline Células de Merkel & 0 & 2 & 2 & 2 & 3 & 0 & 0 & 3 & 3 & 2 & 1 & 5 & 3 & 1 & 5 & 32 \\
\hline Tumores de los anexos & 1 & 2 & 1 & 4 & 2 & 2 & 11 & 4 & 14 & 7 & 17 & 4 & 7 & 12 & 5 & 93 \\
\hline Otras neoplasias & 36 & 39 & 9 & 10 & 8 & 12 & 7 & 13 & 13 & 16 & 24 & 16 & 26 & 40 & 26 & 295 \\
\hline Total & 717 & 702 & 635 & 469 & 494 & 501 & 665 & 828 & 906 & 1.011 & 956 & 871 & 880 & 954 & 1.273 & 11.862 \\
\hline
\end{tabular}

* DFSP: dermatofibrosarcoma protuberans

Fuente. Registro Institucional de Cáncer, Instituto Nacional de Cancerología (datos disponibles a partir del 2003 en http://www.cancer.gov.co/cancer_en_cifr)

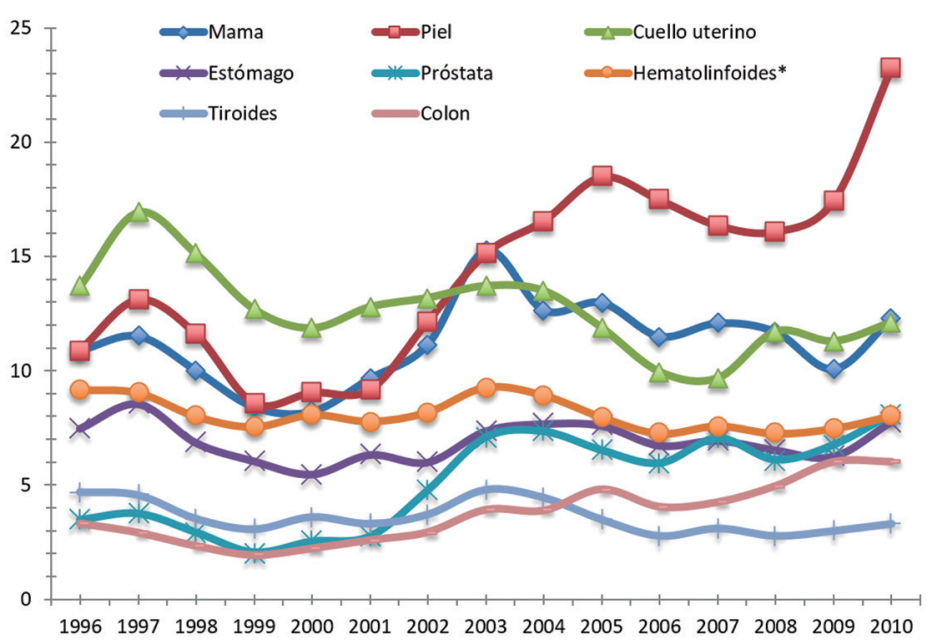

Fuente: Registro Institucional de Cáncer, Instituto Nacional de Cancerología (datos disponibles desde 2003 en: http://www.cancer.gov.co/cancer_en_cifras)

* Hematolinfoides incluye cánceres del sistema hematopoyético, reticuloendotelial y ganglios linfáticos

Figura 1. Casos nuevos de cáncer atendidos en el INC entre 1996 y 2010, comparación de frecuencias por sitio por cada 100 diagnósticos nuevos)

gradualmente la frecuencia de las neoplasias cutáneas en ambos sexos (figura 2), con un pico en los mayores de 75 años, grupo etario en el que se concentró el 30\% de los nuevos casos durante el periodo en mención. Aunque estos hallazgos pueden explicarse por patrones poblacionales de aparición de la enfermedad y patrones de remisión a la institución, concuerdan con lo descrito en otras poblaciones, en las cuales se registra un incremento de las tasas de incidencia a partir de la quinta década y, un pico, durante la séptima y la octava décadas ${ }^{(8)}$.

De los 11.780 casos nuevos de cáncer de la piel, 5.490 (46,6 \%) eran hombres y 6.290 (53,4 \%) mujeres. Los casos nuevos aumentan con la edad y existe una leve diferencia de nuevos diagnósticos, mayor en las mujeres en la mayoría de los grupos etarios. No obstante, otros estudios con estandarización poblacional evidencian un predominio de la incidencia en hombres ${ }^{(9)}$. 
En cuanto al área de interés y la procedencia geográfica, la mayoría de los pacientes atendidos en la institución proceden de Bogotá y de los departamentos del centro del país ${ }^{(10)}$, cercanos a Bogotá; la mayoría pertenece al estrato socioeconómico bajo (I a III), según el tipo de afiliación al Sistema General de Seguridad Social en Salud (SGSSS).

Según los datos que aporta el Registro Institucional del Cáncer del Instituto Nacional de Cancerología, la incidencia estimada del melanoma en Colombia para el período comprendido entre 2002 y 2006, es de 4,6 por 100.000 en mujeres y 4,4 por 100.000 en hombres (tasa ajustada por edad por 100.000 habitantes). Cuando se discriminan los datos por localidad (tabla 2), en Bogotá la tasa aumenta a 6,9 por 100.000 en hombres y a 9.3 por 100.00o en mujeres, así como en Antioquia, 4,5 por 100.000 en mujeres y 6,1 por 100.000 en hombres ${ }^{(7)}$. Estas cifras estimativas son la primera aproximación de los datos de incidencia a nivel nacional para el melanoma maligno, lo que representa un progreso determinante en el conocimiento de la enfermedad.

Por otra parte, para los datos de incidencia en la población colombiana, tradicionalmente se ha tenido en cuenta el Registro Poblacional de Cáncer de Cali que registra desde 1962 los datos de incidencia y de mortalidad del melanoma de la región, el cual evidenció una tasa ajustada por edad de 3,8 por 100.000 en hombres y de 3,1 por 100.000 en mujeres, para el período 2003$2007^{(11)}$.

\section{DISTRIBUCIÓN SEGÚN SUBTIPO HISTOLÓGICO}

Según el subtipo histológico (tabla 1), el carcinoma basocelular fue el más frecuente en el periodo de registro, con un promedio de diagnósticos de 6.371 $(52,7 \%)$ (frecuencia media de 455 casos nuevos por año); seguido por el carcinoma escamocelular, con 2.608 (22,6 \%) casos nuevos (media de 174 por año), y el melanoma maligno, con 1.853 (16,1 \%) (media de 124 por año). Entre los tres, representan el 91,4 \% de los diagnósticos de neoplasias cutáneas.

Otras neoplasias tienen frecuencias más bajas, como los linfomas cutáneos (5,1\%), el sarcoma de Kaposi $(2,7 \%)$ y el dermatofibrosarcoma protuberans $(1,7 \%)$.

\section{MORTALIDAD}

Las cifras de mortalidad están representadas básicamente por las producidas por el melanoma, media de

Tabla 2. Incidencia estimada del melanoma por ente geográfico en Colombia, 2002-2006 (tasa ajustada por edad por 100.000)

\begin{tabular}{|lll|}
\hline \multicolumn{2}{|c|}{$\begin{array}{c}\text { INCIDENCIA ESTIMADA DEL MELANOMA POR ENTE } \\
\text { GEOGÁFICO EN COLOMBIA, 2002-2006 }\end{array}$} \\
\hline \multicolumn{1}{|c}{ Localidad } & Mujeres & Hombres \\
\hline Bogotá & 9.3 & 6.9 \\
\hline Antioquia & 4.5 & 6.1 \\
\hline Valle del Cauca & 3.9 & 5.0 \\
\hline Atlántico & 0.6 & 0.1 \\
\hline Bolívar & 0.4 & 1.2 \\
\hline Norte de Santander & 1.1 & 5.9 \\
\hline Santander & 5.3 & 2.6 \\
\hline Cundinamarca & 8.0 & 4.7 \\
\hline Meta & 9.3 & 5.2 \\
\hline Caldas & 7.0 & 5.5 \\
\hline
\end{tabular}

Fuente: Registro Institucional de Cáncer, Instituto Nacional de Cancerología (http://www.cancer.gov.co/cancer_en_cifras) 


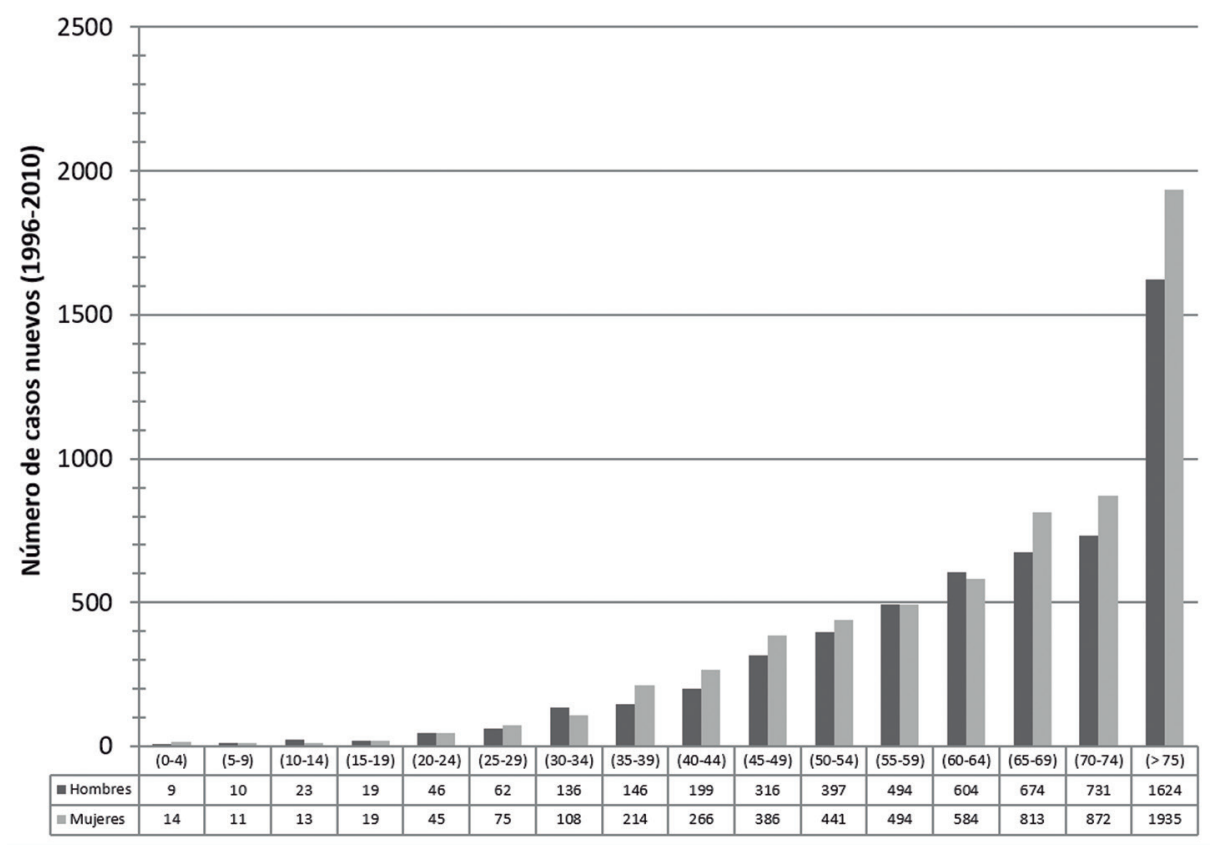

Fuente: Registro Institucional de Cáncer, Instituto Nacional de Cancerología (http://www.cancer.gov.co/cancer_en_cifras)

Figura 2. Casos nuevos de cáncer de piel según sexo y edad, Instituto Nacional de Cancerología, 1996-2010.

16,1 casos por año en el período 2001-2009, sin diferencias según el sexo y con un incremento variable a partir del año 2004.

\section{CONCLUSIÓN}

En el Instituto Nacional de Cancerología, uno de cada cuatro casos nuevos de cáncer atendidos, corresponde a una neoplasia cutánea entre los cuales el cáncer de piel es el más frecuente ${ }^{(7)}$. Aunque los tipos histológicos más frecuentes -carcinoma basocelular, carcinoma escamocelular y melanoma maligno- corresponden a más del $90 \%$ de los diagnósticos, otras neoplasias cutáneas menos frecuentes, como los linfomas, el sarcoma de Kaposi y el dermatofibrosarcoma protuberans, cobran importancia por ser un centro de referencia. Estudios posteriores sobre la etiología y el pronóstico de los subtipos histológicos, asegurarán una mejor comprensión de los fenómenos observados. La información de este reporte puede ser útil como herramienta de consulta, para los dermatólogos y para otros profesionales relacionados con el diagnóstico y tratamiento del cáncer de la piel; también, como un punto de partida para investigaciones posteriores y planeación de procesos de atención. Algunas limitaciones, como la naturaleza retrospectiva de la información, el reporte de dobles patrones histológicos y la confusión residual expresada en la ausencia de medición de otras variables demográficas o clínicas, deben considerarse en la interpretación de estos datos.

Por otro lado, el desarrollo de la investigación sobre alternativas diagnósticas y de tratamiento apropiadas para nuestra población, queda justificado por el volumen importante que representan las neoplasias de piel a nivel institucional y, seguramente, a nivel poblacional.

La estandarización de los modelos de detección, prevención y tratamiento del cáncer de piel, así como el diseño de programas de salud pública, constituyen una necesidad. Por tal motivo, el Instituto Nacional de Cancerología, en el marco del Plan Decenal para el Control del Cáncer, ha diseñado el "Manual para la detección temprana de cáncer de piel” y las "Recomendaciones para la disminución de exposición a rayos ultravioleta”, como parte de una campaña de prevención primaria y secundaria, enfocada a los médicos generales del primer nivel de atención. 


\section{AGRADECIMIENTO}

A los doctores Johana Chávez, Claudia Giraldo, John Suárez, Leonardo Pulido y Xavier Rueda, por sus aportes en la recolección de la información.

\section{REFERENCIAS}

1. Lomas A, Leonardi-Bee J, Bath-Hextall F. A systematic review of worldwide incidence of nonmelanoma skin cancer. Br J Dermatol. 2012;166:106980.

2. Ferlay J, Soerjomataram I, Ervik M, Dikshit R, Eser S, Mathers C, et al. GLOBOCAN 2012 v1.o. Cancer Incidence and Mortality Worldwide: IARC Cancer Base No. 11. Lyon, Fr.: International Agency for Research on Cancer; 2013.

3. Lee DA, Miller SJ. Nonmelanoma skin cancer. Facial Plast Surg Clin North Am. 2009;17:309-24.

4. Shenenberger DW. Cutaneous malignant melanoma: A primary care perspective. Am Fam Physician. 2012;85:161-8.

5. Schmerling R, Loria D, Cinat G, Ramos WE, Cardona AF, Sánchez JL, et al. Cutaneous melanoma in Latin America: The need for more data. Rev Panam Salud Pública. 2011;30:431-8.

6. Nova-Villanueva J, Sánchez-Vanegas G, Porras de Quintana L. Cáncer de piel: perfil epidemiológico de un centro de referencia en Colombia, 20032005. Rev Salud Pública. 2007;9:595-601.

7. Instituto Nacional de Cancerologia [Internet]. Estadisticas. Fecha de consulta: 9 de diciembre de 2015. Disponible en: http://www.cancer.gov.co/ cancer_en_cifras.

8. Kim RH, Armstrong AW. Nonmelanoma skin cancer. Dermatol Clin. 2012;30:125-39.

9. Katalinic A, Kunze U, Schäfer T. Epidemiology of cutaneous melanoma and non-melanoma skin cancer in Schleswig-Holstein, Germany: Incidence, clinical subtypes, tumour stages and localization (epidemiology of skin cancer). Br J Dermatol. 2003;149:1200-6.

10. Pozzobon F, Fierro E, Acosta Á, Carreño A. Características del melanoma cutáneo primario en el Instituto Nacional de Cancerología, 2006-2010. Rev Colomb Cancerol. 2013;17:111-8.

11. Registro Poblacional de Cáncer de Cali. Fecha de consulta: 17 de diciembre de 2011. Disponible en: http://rpcc.univalle.edu.co/es/index.php. 